\title{
Efficacy and safety of first-line erlotinib in elderly patients with advanced non-small cell lung cancer
}

\author{
OFER MERIMSKY ${ }^{1}$, CHI-KIN CHENG ${ }^{2}$, JOSEPH SIU-KIE AU ${ }^{3}$, JOACHIM VON PAWEL ${ }^{4}$ and MARTIN RECK $^{5}$ \\ ${ }^{1}$ Division of Oncology, Tel Aviv Medical Center, Tel-Aviv 64239, Israel; ${ }^{2}$ Department of Oncology, \\ Princess Margaret Hospital, Lai Chi Kok, Hong Kong; ${ }^{3}$ Department of Clinical Oncology, Queen Elizabeth Hospital, \\ Kowloon, Hong Kong, P.R. China; ${ }^{4}$ Department of Oncology, Asklepios Fachkliniken, D-82131 Gauting; \\ ${ }^{5}$ Department of Thoracic Oncology, Krankenhaus Grosshansdorf, D-22927 Grosshansdorf, Germany
}

Received February 16, 2012; Accepted March 21, 2012

DOI: $10.3892 /$ or.2012.1824

\begin{abstract}
TaRceva LUng cancer Survival Treatment (TRUST) was an open-label, phase IV study of advanced non-small cell lung cancer (NSCLC). Patients failing or unsuitable for chemotherapy or radiotherapy received erlotinib $150 \mathrm{mg} /$ day until progression. We examined a subpopulation of elderly patients $(\geq 70$ years) receiving first-line erlotinib $(n=485)$ in TRUST. In this subpopulation, disease control rate $(n=356$ with best response data available) was $79 \%$ (vs. $69 \%$ for the overall TRUST population; $\mathrm{P}<0.0001$ ); median progressionfree survival (PFS) was 4.57 months [95\% confidence interval (CI), 3.68-5.22]; median overall survival (OS) was 7.29 months (95\% CI, 6.27-8.67); and one-year survival, was 36.6\%. PFS and OS were significantly longer in patients developing rash, compared to those without, and in those with good performance status (PS; 0/1), compared to poor PS ( $\geq 2)$. Eighty-seven subpopulation patients (18\%) had an erlotinib-related AE; other than the protocol-defined frequent adverse events (AEs); $4 \%$ had a grade $\geq 3$ erlotinib-related AE, $7 \%$ had an erlotinibrelated serious $\mathrm{AE}$. In the subpopulation, dose reductions were required in $27 \%$, most (97\%) were reductions to $100 \mathrm{mg}$ / day; treatment was discontinued in $10 \%$, and one death was associated with treatment-related toxicity $(<1 \%)$. Erlotinib was effective and well-tolerated and may be considered for elderly patients with advanced NSCLC who are unsuitable for standard first-line chemotherapy or radiotherapy.
\end{abstract}

\section{Introduction}

The incidence of non-small cell lung cancer (NSCLC) increases with age, with $60 \%$ of cases arising in patients over 60 years of age, and 30-40\% in patients of 70 years or older

Correspondence to: Dr Ofer Merimsky, Division of Oncology, Tel Aviv Medical Center, 6 Weizmann Street, Tel-Aviv 64239, Israel E-mail: oferm@tasmc.health.gov.il

Key words: chemotherapy, elderly, erlotinib, first-line, phase IV, non-small cell lung cancer
$(1,2)$. In developed countries the median age of diagnosis for advanced disease is 68 years (3), but this has increased notably over the last 3 decades. Consequently, the age of the patient is often a major factor for physicians considering treatment options for patients with advanced NSCLC. Elderly patients are often denied therapy, prematurely discontinued and excluded from clinical trials because of the perception that they are less able to tolerate cytotoxic chemotherapy than younger patients, and are more likely to suffer toxic effects that adversely affect their quality of life (4). Physicians may be concerned that age-related impairment in renal or hepatic function could exacerbate the toxic effects of chemotherapy, and that the presence of comorbidities could reduce the capacity of their elderly patients to tolerate such effects. While it is true that the incidence of age-related organ dysfunction and the development of comorbid conditions increase abruptly between 70 and 75 years of age (5), the clinical significance of the relationship between age and comorbid conditions is complex in patients with cancer (6) and it has been suggested that chronological age is not a valid criterion on which to base treatment decisions in NSCLC (7). Clinical trials that have investigated the effects of cytotoxic chemotherapy in unselected elderly patients have reported modest improvements in outcomes (8-11), while an analysis of two large, randomised trials of adjuvant/palliative chemotherapy for NSCLC found that the age of the patient did not influence overall survival (OS), but that the presence of comorbid conditions was linked with poorer survival (7).

New targeted therapies for NSCLC, such as the human epidermal growth factor receptor (EGFR) tyrosine-kinase inhibitor, erlotinib (Tarceva ${ }^{\circledR}$ ), combine therapeutic efficacy with a relatively benign toxicity profile compared with cytotoxic chemotherapy, and so represent attractive new therapeutic options for the treatment of elderly patients with NSCLC (12). Erlotinib has been demonstrated to significantly prolong OS vs. placebo in previously-treated patients with NSCLC $(n=731)$ (13), with the improvement in survival benefit being apparent in a broad range of patient subtypes. Erlotinib is now approved in more than 80 countries for the treatment of patients who have received at least one line of chemotherapy.

The TaRceva LUng cancer Survival Treatment (TRUST) study was a large, open-label, phase IV study of erlotinib, designed to allow access to erlotinib monotherapy for patients 
with advanced stage IIIB/IV NSCLC who had previously failed, or were considered unsuitable to receive, standard chemotherapy or radiotherapy, and were ineligible for other erlotinib trials (14). A total of 6,665 patients were enrolled in the study, from 513 centres across 51 countries, and recruitment ceased on 31 May, 2007. The cut-off date for the final analysis was 17 April, 2009. The findings of the study confirmed the favourable efficacy and safety profile of erlotinib in a large heterogeneous population of NSCLC patients; the 1-year survival rate was $37.7 \%$, and median OS and progressionfree survival (PFS) were 7.9 and 3.25 months, respectively (14). The large size of the study database made it feasible to evaluate outcomes among the large number of older patients ( $\geq 70$ years) who participated; this report describes outcomes among elderly patients who received erlotinib as their first line therapy.

\section{Patients and methods}

Patients. Eligible patients had histologically- or cytologicallyconfirmed, unresectable, stage IIIB/IV NSCLC. This analysis was limited to patients who had received no previous therapies for their NSCLC (systemic chemotherapy or radiotherapy) because they were ineligible to receive such treatment. Patients of both Asian and non-Asian ethnicity were included in the analysis. Patients were unsuitable for other erlotinib trials. Other eligibility criteria included: age $\geq 18$ years, Eastern Cooperative Oncology Group (ECOG) performance status (PS) 0-3; adequate haematological, renal and hepatic function; estimated life expectancy of $\geq 12$ weeks [full details of eligibility criteria and other methodological details were described by Reck et al (14)]. Key exclusion criteria included: evidence of unstable systemic disease; prior treatment with anti-EGFR agents; any previous malignancies within the last 5 years (other than cervical carcinoma in situ or skin cancer that underwent successful treatment); untreated brain metastases (newly diagnosed or pre-existing) or spinal cord compression; and any significant ophthalmological abnormalities.

The study complied with the Declaration of Helsinki and Good Clinical Practice guidelines. Informed consent was obtained from all patients, and the protocol was approved at all centres by appropriate ethics committees.

Study treatment. Oral erlotinib was administered once daily at a dose of $150 \mathrm{mg}$ to all patients, until unacceptable toxicity, disease progression or death. Dose interruption or reduction (in $50 \mathrm{mg}$ decrements) was permitted in the event of treatmentrelated adverse events (AEs).

Clinical assessments. Outcomes included: best response (as assessed by the investigators); PFS; OS; safety and tolerability [including incidence and grade of erlotinib-related rash, serious AEs (SAEs), treatment-related SAEs and AEs leading to treatment withdrawal]. Clinical and laboratory assessments were conducted at baseline, then every 4 weeks throughout the study. Tumour response was assessed using Response Evaluation Criteria in Solid Tumours (RECIST) (15), at least every 2 months. Tumour responses were confirmed by a second assessment, 4 weeks after the initial determination of response. Safety and tolerability evaluations were assessed and
Table I. Baseline demographic and clinical characteristics for elderly patients ( $\geq 70$ years) who received first-line erlotinib $(n=485)$, and for the overall TRUST population $(n=6,580)$.

\begin{tabular}{|c|c|c|}
\hline Characteristics & $\begin{array}{c}\text { TRUST } \\
\text { first-line } \\
\text { elderly } \\
\text { population }\end{array}$ & $\begin{array}{c}\text { TRUST } \\
\text { overall } \\
\text { population (14) }\end{array}$ \\
\hline Median age, years (range) & $77(70-91)$ & $63(19-91)$ \\
\hline \multicolumn{3}{|l|}{ Gender, n (\%) } \\
\hline Male & $260(54)$ & $3,974(60)$ \\
\hline Female & $225(46)$ & $2,606(40)$ \\
\hline \multicolumn{3}{|l|}{ Ethnic origin, $\mathrm{n}(\%)$} \\
\hline Caucasian/white & $399(82)$ & 5057 (77) \\
\hline Black & $2(<1)$ & $29(<1)$ \\
\hline Asian & $76(16)$ & $1,345(20)$ \\
\hline Other & $8(2)$ & $149(2)$ \\
\hline \multicolumn{3}{|l|}{ ECOG PS, n (\%) } \\
\hline 0 & $72(15)$ & $1,473(22)$ \\
\hline 1 & $231(48)$ & $3,504(53)$ \\
\hline 2 & $139(29)$ & $1,235(19)$ \\
\hline 3 & $43(9)$ & $360(5)$ \\
\hline No data & $0(0)$ & $8(<1)$ \\
\hline \multicolumn{3}{|l|}{ Stage, n (\%) } \\
\hline Stage IIIB & $140(29)$ & $1,376(21)$ \\
\hline Stage IV & $338(70)$ & $5,185(79)$ \\
\hline Other & $6(1)$ & $15(<1)$ \\
\hline No data & $1(<1)$ & $4(<1)$ \\
\hline \multicolumn{3}{|l|}{ Histology, n (\%) } \\
\hline Adenocarcinoma & $243(50)$ & $3,590(55)$ \\
\hline Bronchoalveolar carcinoma & $61(13)$ & $372(6)$ \\
\hline Large-cell carcinoma & $20(4)$ & $382(6)$ \\
\hline Squamous-cell carcinoma & $106(22)$ & $1,552(24)$ \\
\hline Other & $55(11)$ & $681(10)$ \\
\hline No data & $0(0)$ & $3(<1)$ \\
\hline \multicolumn{3}{|l|}{ Smoking status, n (\%) } \\
\hline Non-smoker & $158(33)$ & $2,004(30)$ \\
\hline Current or former smoker & $327(67)$ & 4,567 (69) \\
\hline No data & $0(0)$ & $9(<1)$ \\
\hline
\end{tabular}

ECOG PS, Eastern Cooperative Oncology Group performance status; TRUST, TaRceva LUng cancer Survival Treatment.

graded using National Cancer Institute Common Terminology Criteria for Adverse Events version 3.0. Treatment-related AEs were reported if they were not included on a list of pre-specified AEs defined in the study protocol (i.e., rash, pruritus, dry skin, diarrhoea, nausea, vomiting, stomatitis, abdominal pain, fatigue, dyspnea, cough, anorexia, infection, conjunctivitis and keratoconjunctivitis sicca).

Statistical analysis. PFS was determined from the date of erlotinib initiation until the date of first documented progres- 
Table II. Best response rates with erlotinib for elderly patients ( $\geq 70$ years) who received first-line erlotinib ( $\mathrm{n}=356$ ), and for the overall TRUST population $(n=5,394)$.

Best response to therapy in patients with data available, $\mathrm{n}(\%)$

TRUST first-line elderly population

TRUST overall population (14)

\begin{tabular}{lcc}
\hline Complete response & $3(<1)$ & $45(<1)$ \\
Partial response & $47(13)$ & $668(12)$ \\
Stable disease & $231(65)$ & $2,992(55)$ \\
Progressive disease & $55(15)$ & $1,483(27)$ \\
Not evaluable & $20(6)$ & $206(4)$ \\
Overall response rate $^{\mathrm{a}}(\%)$ & 14 & 13 \\
Disease control rate $^{\mathrm{b}}(\%)$ & 79 & 69
\end{tabular}

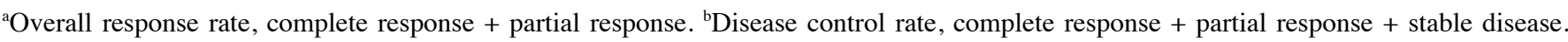
TRUST, TaRceva LUng cancer Survival Treatment.
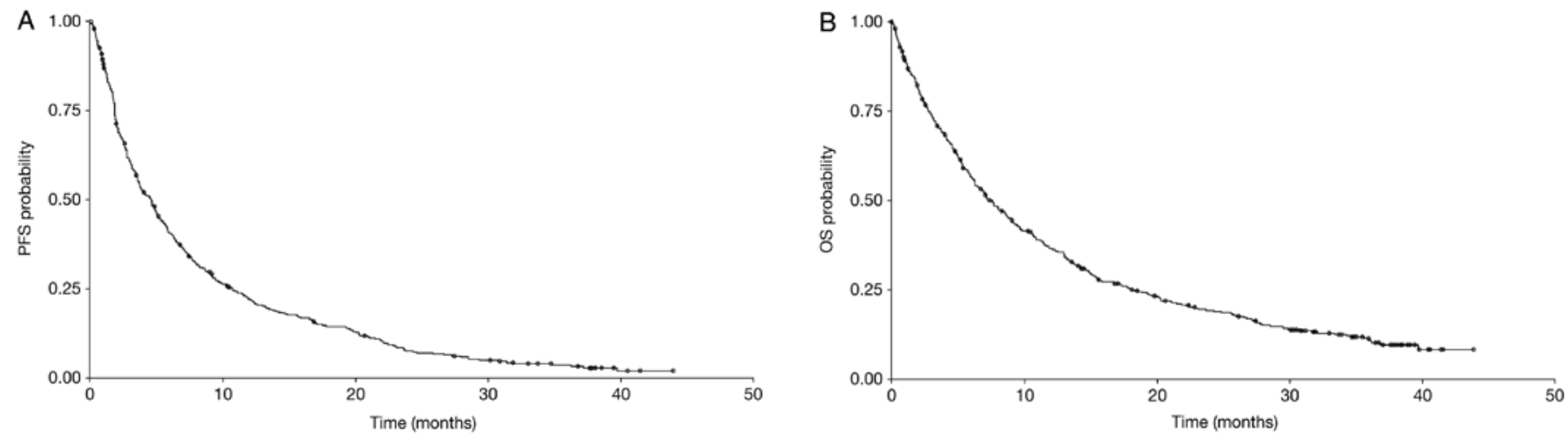

Figure 1. (A) PFS and (B) OS in elderly patients ( $\geq 70$ years) receiving first-line erlotinib $(n=485)$.

sion according to RECIST objective tumour assessment, or until the date of death for any reason in the absence of disease progression. OS was determined from the date of initiation of erlotinib until the date of death from any cause.

\section{Results}

Patients. The intent-to-treat population $(\mathrm{n}=485)$ included all patients $\geq 70$ years of age who had received at least one dose of first-line erlotinib and for whom clinical data (from submitted case report forms) had been entered in the study database by the cut-off date of 17 April, 2009. Demographic and disease characteristics at baseline for the 'first-line erlotinib/elderly' group and for the overall TRUST population are shown in Table I. Seventy-six of the 485 'first-line erlotinib/elderly' patients (16\%) were of Asian ethnicity, just over half (54\%) were male, and two-thirds (67\%) were current or former smokers. Fifty percent had adenocarcinoma and $22 \%$ had squamous-cell carcinoma. Seventy percent had stage IV disease (a slightly smaller percentage than in the overall TRUST population (79\%)] and $38 \%$ were ECOG PS 2/3, compared with $24 \%$ of the total population.

Response and survival. Best response data for erlotinib (Table II) were available for 356 of the 'first-line erlotinib/ elderly' group, among whom the overall response rate was $14 \%$, compared with $13 \%$ for the overall TRUST population. The disease control rate for erlotinib was $79 \%$ (compared with $69 \%$ for the overall TRUST population; $\mathrm{P}<0.0001)$. Median PFS for the 'first-line erlotinib/elderly' group was 4.57 months [95\% confidence interval (CI), 3.68-5.22] (Fig. 1A), and median OS was 7.29 months (95\% CI, 6.27-8.67) (Fig. 1B), while the one-year survival rate was $36.6 \%$.

Median PFS and OS were both significantly longer for patients who developed erlotinib-related rash (Fig. 2). For patients with rash, median PFS was 6.08 months (95\% CI, 5.03-7.10) vs. 2.04 months (95\% CI, 1.64-2.92) for those without rash [hazard ratio [HR) $0.36 ; \mathrm{P}<0.001$ ], and median OS was 10.91 months (95\% CI, 9.10-13.21) and 3.12 months (95\% CI, 2.17-4.96), respectively (HR 0.30; P<0.0001).

Survival parameters were also determined separately for elderly patients of Asian ethnicity $(n=76)$ and non-Asian ethnicity $(\mathrm{n}=409)$ receiving first-line erlotinib. Median PFS was 4.86 months (95\% CI, 3.25-9.13) for Asian patients and 4.24 months (95\% CI, 3.58-5.03) for non-Asian patients (HR $1.15 ; \mathrm{P}=0.27)$, and median OS was 9.69 months $(95 \% \mathrm{CI}$, 6.44-11.89) and 7.19 months (95\% CI, 6.14-8.54), respectively (HR 1.15; $\mathrm{P}=0.31$ ).

Elderly patients with poor PS ( $n=139$ for PS 2 and $n=43$ for PS 3) had worse survival outcomes than those with good 

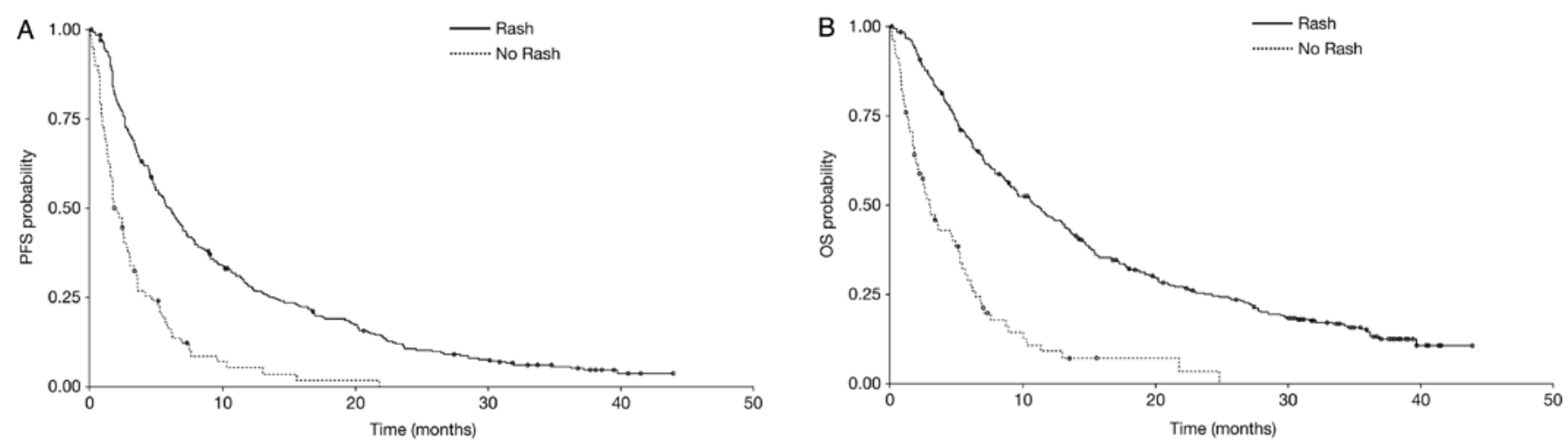

Figure 2. (A) PFS and (B) OS for elderly patients ( $\geq 70$ years) receiving first-line erlotinib, according to the occurrence of erlotinib-related rash.

Table III. Tolerability of erlotinib therapy in elderly patients ( $\geq 70$ years) treated in the first-line setting $(n=485)$ and in the overall population in the TRUST study $(\mathrm{n}=6,580)$.

AEs, n (\%)

TRUST first-line

elderly population
TRUST

overall population (14)
Patients with at least one treatment-related $\mathrm{AE}^{\mathrm{a}}$

Patients with at least one treatment-related SAE

Patients who discontinued study due to treatment-related AEs

Patients who died due to a treatment-related AE

$\begin{array}{cc}87(18) & 799(12) \\ 35(7) & 274(4) \\ 50(10) & 337(5) \\ 2(<1) & 24(<1)\end{array}$

${ }^{\mathrm{a}}$ Other than the pre-specified events defined in the protocol. AE, adverse event; SAE, serious adverse event; TRUST, TaRceva LUng cancer Survival Treatment.

Table IV. Incidence of specific SAEs in elderly patients ( $\geq 70$ years) receiving first-line erlotinib $(n=485)$ vs. the overall TRUST population $(\mathrm{n}=6,580)$.

\begin{tabular}{|c|c|c|c|c|}
\hline Patients with at least one treatment-related SAE & $35(7)$ & $29(6)$ & $274(4)$ & $209(3)$ \\
\hline Gastrointestinal disorders & $15(3)$ & $11(2)$ & $121(2)$ & $89(1)$ \\
\hline Diarrhoea & $9(2)$ & $8(2)$ & $67(1)$ & $49(<1)$ \\
\hline Nausea & $3(<1)$ & $2(<1)$ & $24(<1)$ & $11(<1)$ \\
\hline General disorders and administrative site conditions & $2(<1)$ & $2(<1)$ & $26(<1)$ & $18(<1)$ \\
\hline Hepatobiliary disorders & $2(<1)$ & $2(<1)$ & $5(<1)$ & $5(<1)$ \\
\hline Infections and infestations & $2(<1)$ & $2(<1)$ & $22(<1)$ & $16(<1)$ \\
\hline Metabolism and nutrition disorders & $7(1)$ & $4(<1)$ & $27(<1)$ & $18(<1)$ \\
\hline Dehydration & $4(<1)$ & $2(<1)$ & $15(<1)$ & $10(<1)$ \\
\hline Respiratory, thoracic and mediastinal disorders & $3(<1)$ & $3(<1)$ & $37(<1)$ & $30(<1)$ \\
\hline Pneumonitis & $2(<1)$ & $2(<1)$ & $6(<1)$ & $6(<1)$ \\
\hline Skin and subcutaneous tissue disorders & $9(2)$ & $7(1)$ & $40(<1)$ & $31(<1)$ \\
\hline Rash & $8(2)$ & $7(1)$ & $31(<1)$ & $26(<1)$ \\
\hline
\end{tabular}

ancludes SAEs reported in $>1$ patient; no grade 5 SAEs were reported in the first-line elderly population, compared with 11 grade 5 SAEs in the overall population. SAE, serious adverse event; TRUST, TaRceva LUng cancer Survival Treatment. 
PS ( $\mathrm{n}=303)$, median PFS was 5.58 months (95\% CI, 4.73-6.70) for PS 0/1 vs. 3.15 months (95\% CI, 2.56,4.14) for PS 2 and 1.81 months (95\% CI, 1.38-2.73) for PS 3. Similar results were seen with median OS, those with PS 0/1 had median OS of 10.38 months (95\% CI, 8.54-12.29) while those with a PS of 3 had median OS of just 2.07 months (95\% CI, 1.45-3.68).

Toxicity. Safety data were available for all 485 'first-line erlotinib/elderly' patients, 325 of whom (67\%) had at least one AE (Table III). Eighty-seven patients (18\%) had an erlotinib-related AE (other than the pre-specified AEs defined in the study protocol) and 20 patients (4\%) had a grade $\geq 3$ erlotinib-related AE [compared with 173 patients (3\%) in the overall TRUST population]. Seven percent of patients had a treatment-related SAE, compared with $4 \%$ of the overall TRUST population (Table IV). Dose reductions with erlotinib occurred in $27 \%$ of the 'first-line erlotinib/elderly' group; most of these (97\%) were reductions to $100 \mathrm{mg} /$ day. By comparison, in the overall TRUST population, $17 \%$ of patients received a reduced dose of erlotinib, and $96 \%$ of these were reductions to $100 \mathrm{mg} / \mathrm{day}$. Erlotinib-related AEs led to treatment discontinuation in 50 of the 'first-line erlotinib/elderly' group (10\%), while two deaths were associated with treatment-related toxicity $(<1 \%)$. In the overall TRUST population, 337 patients $(5 \%)$ discontinued treatment due to erlotinib-related AEs and 24 deaths $(<1 \%)$ were reported due to treatment toxicity.

Biomarkers. Of the 485 patients included in this analysis, EGFR mutation status was known for just 18 patients: 16 had wild-type tumours and two had EGFR mutation-positive tumours. Both patients with mutation-positive status were female and Caucasian, with stage IV disease and an ECOG PS of 2. Patient 1 was 80 years old and was a never smoker with non-adenocarcinoma histology; she had PFS and OS of 8.54 months. Patient 2 was a 78 -year-old former/current smoker with adenocarcinoma; she had an impressive PFS of 31.9 months and OS of 41.6 months.

\section{Discussion}

The goals of first-line chemotherapy for elderly NSCLC patients ( $\geq 65$ years old) are similar to those for younger patients, i.e., to extend survival and relieve cancer-related symptoms, but relatively few randomised controlled studies have specifically investigated elderly patients with NSCLC. The first such trial was the Elderly Lung Cancer Vinorelbine Italian Study, in which single-agent vinorelbine was reported to improve quality of life and survival vs. supportive care alone in patients older than 70 years of age (median survival 27 vs. 21 weeks, $P=0.04$ ) (16). Five of 71 patients discontinued treatment due to severe toxic events (grade $3 / 4$ constipation, grade 2 heart toxicity), and grade 4 leucopenia occurred in 4 patients but did not lead to discontinuation.

In the first randomised phase III trial with taxane monotherapy in elderly patients with advanced NSCLC, treatment with docetaxel achieved a statistically significant improvement in PFS (5.5 vs. 3.1 months) and response rate (22.7\% vs. 9.9\%) compared with vinorelbine (17). OS favoured docetaxel (10.3 vs. 6.4 months) but the difference was not statistically significant (HR 0.78; $\mathrm{P}=0.65$ ). Grade $3 / 4$ neutropenia occurred in more patients on docetaxel than vinorelbine $(\mathrm{P}=0.031)$, but there was no difference in the incidence of grade $3 / 4$ febrile neutropenia and infection.

Combination chemotherapy has also been evaluated in a small number of trials involving elderly populations. An interim analysis of a trial comparing gemcitabine plus vinorelbine with vinorelbine alone suggested a significant advantage for the combination arm (median survival of 7 vs. 4.5 months, respectively) (18). The combination regimen resulted in grade 3/4 neutropenia and thrombocytopenia in 38 and $13 \%$ of patients, respectively, higher incidences than seen with vinorelbine alone ( 28 and $8 \%$, respectively). These findings were challenged by the Multicenter Italian Lung Cancer in the Elderly Study, a large, randomised phase III trial involving 698 NSCLC patients with a median age of 74 years (8). In this trial the combination of vinorelbine and gemcitabine was no more effective than single-agent vinorelbine or gemcitabine (median survival was 36 weeks for vinorelbine, 28 weeks for gemcitabine and 30 weeks for the combination). Moreover, the combination was slightly more toxic, although quality of life was similar across the three treatment arms, and the authors concluded that until further studies showed a clear benefit for combination therapy, the recommended treatment for elderly patients with advanced NSCLC should be single-agent chemotherapy. This was supported by another phase III study that compared the efficacy of monotherapy vs. combination chemotherapy in elderly patients ( $>65$ years of age) and those with poor PS (19). Eighty percent of patients enrolled were $>65$ years of age, and the results showed that combination chemotherapy led to a modest improvement in time to progression (4.8 vs. 2.9 months; $\mathrm{P}=0.004)$ without an effect on OS (5.5 vs. 5.1 months; $\mathrm{P}=0.65$ ). Grade 3/4 myelosuppression was significantly more common with combination therapy.

The results of a retrospective analysis of the ECOG 4599 trial [carboplatin, paclitaxel and bevacizumab (PCB) vs. carboplatin and paclitaxel alone (PC)], on the basis of age have also been reported (20). Twenty-six percent of patients $(n=224)$ enrolled in the trial were $>70$ years of age; 113 were randomised to $\mathrm{PC}$ and 111 to $\mathrm{PCB}$. Compared with younger patients, the elderly group had more toxicity with PCB $(87 \%$ had grade 3 toxicity compared with $70 \%$ of the younger group; $\mathrm{P}<0.001)$. Significant toxicities included grade 4 neutropenia, gastrointestinal bleed, proteinuria, muscle weakness, neuropathy and dizziness. There was a superior response rate and a non-significant trend towards improved PFS in the elderly group, but no difference in OS ( $\mathrm{PCB}=11.3$ months; $\mathrm{PC}=12.1$ months; $\mathrm{P}=0.4$ ).

Elderly patients are more likely than younger patients to suffer from age-related impairments in organ function, as well as other comorbidities and pre-existing medical conditions. Consequently, it is essential that NSCLC treatments considered for use in elderly patients should not induce unacceptable levels of toxicity or impair patients' quality of life. In previously-treated patients with NSCLC (13), it has been reported that erlotinib monotherapy achieved improvements in survival compared with placebo, among all patient subgroups evaluated, including those aged $\geq 60$ years.

The current findings demonstrate that erlotinib is well tolerated as a first-line treatment for elderly patients with 
advanced NSCLC. Efficacy outcomes for elderly patients in the first-line setting were at least comparable to those for elderly patients in previous erlotinib trials. The median PFS was somewhat longer for the TRUST 'first-line erlotinib/ elderly' group (4.57 months) than for the overall TRUST population (3.25 months). In a phase II trial of first-line erlotinib in patients aged $\geq 70$ years (12), the median time to progression was reported as 3.5 months. Although the data are not directly comparable between the BR.21 trial and the current analysis, because BR.21 examined the use of erlotinib in the second-/ third-line setting, PFS for elderly patients in the BR.21 study was 3 months (21). The disease control rate for the TRUST 'first-line erlotinib/elderly' group (79\%) was significantly higher than for the overall TRUST population $(69 \% \mathrm{P}<0.0001)$ and the median OS for the 'first-line erlotinib/elderly' patients (7.29 months) was similar to that for the overall TRUST population (7.9 months).

Tolerability is a key consideration for medications that are to be used for the treatment of elderly patients receiving therapy for advanced NSCLC. It is therefore important to recognise that in this analysis of elderly patients receiving erlotinib in the first-line setting, no new safety signals were identified. The most common AE seen with erlotinib is skin rash, but this is usually mild and manageable and also has potential as a surrogate marker of therapeutic efficacy. As reported in previous studies of erlotinib (22), the current analysis found that both PFS and OS were significantly longer in patients who developed rash than in those who did not.

The incidence of erlotinib-related AEs and SAEs was slightly higher in this subgroup population than in the overall TRUST population, but the differences were not significant. Dose reductions and discontinuations due to toxicity were slightly more common in the 'first-line erlotinib/elderly' population than in the overall TRUST population, but this may be because elderly patients tend to have reduced organ function compared with younger patients, and so may have higher exposure to erlotinib. The presence of co-morbidities may also have exacerbated any adverse reactions to erlotinib.

Elderly patients are also more likely to have reduced PS, which may affect response to treatment. Results presented from the Tarceva Or Placebo In Clinically Advanced Lung cancer (TOPICAL) study of erlotinib in NSCLC patients with poor PS in the UK suggest that this factor may limit the benefit obtained from erlotinib treatment in some subgroups, particularly in male patients (23). The results of this analysis demonstrate that PS is inversely related to survival, with those patients who have poor PS having a significantly shorter median PFS and OS than those with good PS. It is likely that this difference would be observed regardless of treatment, however, as these are the patients most likely to experience rapid progression and deterioration before they have a chance to benefit from treatment.

In conclusion, erlotinib was found to be effective and well tolerated as a first-line treatment for elderly patients involved in the TRUST study. Erlotinib should be considered for the treatment of elderly patients with advanced NSCLC who are unable to receive standard chemotherapy or radiotherapy.

\section{Acknowledgements}

The TRUST study was supported by funding from F. Hoffmann-La Roche Ltd., which was involved in the study design, collection, analysis and interpretation of the data. Support for third party writing assistance for this manuscript was provided by F. Hoffmann-La Roche Ltd. Ofer Merimsky has received payment for a leadership position/ advisory role from Medison and Pfizer. He has also received honoraraia from Eli Lilly and research funding from F. Hoffmann-La Roche. Martin Reck has received payment for consultancy on advisory boards from F. Hoffmann-La Roche Ltd., Lilly, Pfizer, BMS and AstraZeneca, as well as honoraria for lectures from F. Hoffmann-La Roche Ltd., Lilly, Merck and AstraZeneca.

\section{References}

1. Weir HK, Thun MJ, Hankey BF, et al: Annual report to the nation on the status of cancer, 1975-2000, featuring the uses of surveillance data for cancer prevention and control. J Natl Cancer Inst 95: 1276-1299, 2003.

2. Gridelli C, Perrone F and Monfardini S: Lung cancer in the elderly. Eur J Cancer 33: 2313-2314, 1997.

3. Bunn PA Jr and Lilenbaum R: Chemotherapy for elderly patients with advanced non-small cell lung cancer. J Natl Cancer Inst 95: 341-343, 2003.

4. Alam N, Shepherd FA, Winton T, et al: Compliance with postoperative adjuvant chemotherapy in non-small cell lung cancer: an analysis of National Cancer Institute of Canada and intergroup trial JBR 10 and a review of the literature. Lung Cancer 47: 385-394, 2005.

5. Balducci L: Geriatric oncology: challenges for the new century. Eur J Cancer 36: 1741-1754, 2000.

6. Balducci L: Geriatric oncology. Crit Rev Oncol Hematol 46: 211-220, 2003.

7. Asmis TR, Ding K, Seymour L, et al, National Cancer Institute of Canada Clinical Trials Group: Age and comorbidity as independent prognostic factors in the treatment of non-small cell lung cancer: a review of National Cancer Institute of Canada Clinical Trials Group trials. J Clin Oncol 26: 54-59, 2008.

8. Gridelli C, Perrone F, Gallo C, et al: Chemotherapy for elderly patients with advanced non-small cell lung cancer: the Multicenter Italian Lung Cancer in the Elderly Study (MILES) phase III randomized trial. J Natl Cancer Inst 95: 362-372, 2003.

9. Boukovinas I, Souglakos J, Hatzidaki D, et al: Docetaxel plus gemcitabine as front-line chemotherapy in elderly patients with lung adenocarcinomas: a multicenter phase II study. Lung Cancer 63: 77-82, 2009.

10. Gridelli C, De Maio E, Barbera S, et al: The MILES-2G phase 2 study of single-agent gemcitabine with prolonged constant infusion in advanced non-small cell lung cancer elderly patients. Lung Cancer 61: 67-72, 2008.

11. Pallis AG, Polyzos A, Boukovinas I, et al: Pooled analysis of elderly patients with non-small cell lung cancer treated with front line docetaxel/gemcitabine regimen: the Hellenic Oncology Research Group experience. J Thorac Oncol 3: 505-510, 2008.

12. Jackman DM, Yeap BY, Lindeman NI, et al: Phase II clinical trial of chemotherapy-naive patients $>$ or $=70$ years of age treated with erlotinib for advanced non-small cell lung cancer. J Clin Oncol 25: 760-766, 2007.

13. Shepherd FA, Rodrigues Pereira J, Ciuleanu T, et al: National Cancer Institute of Canada Clinical Trials Group. Erlotinib in previously treated non-small-cell lung cancer. N Engl J Med 353: $123-132,2005$.

14. Reck M, van Zandwijk N, Gridelli C, et al: Erlotinib in advanced non-small cell lung cancer: efficacy and safety findings of the global phase IV Tarceva Lung Cancer Survival Treatment study. J Thorac Oncol 5: 1616-1622, 2010.

15. Therasse P, Arbuck SG, Eisenhauer EA, et al: New guidelines to evaluate the response to treatment in solid tumors. J Natl Cancer Inst 92: 205-216, 2000. 
16. The Elderly Lung Cancer Vinorelbine Italian Study Group: Effects of vinorelbine on quality of life and survival of elderly patients with advanced non-small-cell lung cancer. J Natl Cancer Inst 91: 66-72, 1999

17. Kudoh S, Takeda K, Nakagawa K, et al: Phase III study of docetaxel compared with vinorelbine in elderly patients with advanced non-small-cell lung cancer: results of the West Japan Thoracic Oncology Group Trial (WJTOG 9904). J Clin Oncol 24: 3657-3663, 2006.

18. Frasci G, Lorusso V, Panza N, et al: Gemcitabine plus vinorelbine yields better survival outcome than vinorelbine alone in elderly patients with advanced non-small cell lung cancer. A Southern Italy Cooperative Oncology Group (SICOG) phase III trial. Lung Cancer 34: S65-S69, 2001.

19. Hainsworth JD, Spigel DR, Farley C, et al: Weekly docetaxel versus docetaxel/gemcitabine in the treatment of elderly or poor performance status patients with advanced non small cell lung cancer: a randomized phase 3 trial of the Minnie Pearl Cancer Research Network. Cancer 110: 2027-2034, 2007.
20. Ramalingam SS, Dahlberg SE, Langer CJ, et al: Outcomes for elderly, advanced-stage non small-cell lung cancer patients treated with bevacizumab in combination with carboplatin and paclitaxel: analysis of Eastern Cooperative Oncology Group Trial 4599. J Clin Oncol 26: 60-65, 2008.

21. Wheatley-Price P, Ding K, Seymour L, et al: Erlotinib for advanced non-small cell lung cancer in the elderly: an analysis of the National Cancer Institute of Canada Clinical Trials Group Study BR.21. J Clin Oncol 26: 2350-2357, 2008.

22. Wacker B, Nagrani T, Weinberg J, et al: Correlation between development of rash and efficacy in patients treated with the epidermal growth factor receptor tyrosine kinase inhibitor erlotinib in two large phase III studies. Clin Cancer Res 13: 3913-3921, 2007.

23. Lee S, Rudd R, Khan I, et al: TOPICAL: randomized phase III trial of erlotinib compared with placebo in chemotherapy-naïve patients with advanced non-small cell lung cancer (NSCLC) and unsuitable for first-line chemotherapy. J Clin Oncol 28 (Suppl 15): 7504, 2010. 